\title{
ANALYSIS OF ROOT SYSTEM ARCHITECTURE AFFECTED BY SWARMING BEHAVIOR
}

\author{
Songyang LI*, Wenqi YU, Xiaodong LIU, Miao WANG \\ Henan University of Engineering, China
}

Received: January 2020; Accepted: March 2020

\begin{abstract}
The root system architecture (RSA) displays complex morphological characteristics because of diverse root growth behaviors. Recent studies have revealed that swarming behavior among roots is particularly important for RSA to adapt to environmental stimuli. However, few models are proposed to simulate RSA based on swarming behavior of roots. To analyze plasticity of RSA affected by swarming behavior, we propose viewing it as a swarm of single roots. A swarming behavior model is proposed by considering repulsion, alignment, and preference of individual single roots. Then, the swarming behavior model is integrated into a simple and generic RSA model (called ArchiSimple). Lastly, characteristics of RSA affected by swarming behavior model and non-swarming behavior model are compared and analyzed under three different virtual soil sets. The characteristics of RSA (such as primary root length, lateral root length, lateral roots, and resource uptake) are significantly promoted by swarming behavior. Root system distributions can also be greatly affected by swarming behavior. These results show that root foraging and exploration in soil can be regarded as collective behavior of individual single root.
\end{abstract}

Key words: swarming behavior; root growth strategies; soil environment; functional-structural root modeling; root plasticity

\section{INTRODUCTION}

The root system architecture (RSA) is a very important component of a plant's strategy to survive, representing the adaptation to the available nutrients in the soil (McCleery et al. 2017). Root systems have developed complex architectures to optimally exploit soil nutrients (Barlow \& Fisahn 2013). In the process of the interaction between a root system and soil, the root system can acclimate to the environment through architectural changes that act at the root-type level changes (Rellán-Álvarez et al. 2016).

Previous studies have shown that a root system can perform plastic directional growth in response to a directional stimulus. For example, a root system is capable of reorienting itself toward the position of nutrient-rich areas in response to gradients of water and chemicals (Cassab et al. 2013; Hodge 2004).
This phenomenon is known as tropism. It enables a root system to hunt around its surroundings for an adequate supply of water and other nutrients (Gilroy 2008). Root systems can monitor soil environmental changes and then grow toward appropriate resource areas. This ability is very important, because it allows exploiting the root system surroundings. It is also very critical for the plasticity of the root architecture. In addition to tropism, root systems can also use different growth and branching strategies to affect the root architecture. For example, a root system may proliferate when it encounters a nutrient-rich zone. The absorptive surface of the root system can increase to adapt to nutrient availability (Kiba and Krapp 2016). The branching density is improved under adequate nutrient availability. The emergence of branching is suppressed when the surrounding region is lacking in nutrients (Zhang \& Forde 1998). 
When a root system grows through heterogeneous patches of high and low nutrient levels, a substantial amount of branching emerges in the high nutrient zone (Giehl et al. 2012). Lateral and primary root elongations have very different responses to low or high phosphate and nitrate availability in the soil (Tian et al. 2014). In addition to the above strategies, hierarchical modification of the growth rate between a lateral root and a parent root (on which the lateral root is borne) has also been observed as a means to adapt to nutrient availability (Henke et al. 2014). For example, a root system has thin and short lateral roots in nutrient-poor zones, while it tends to develop thicker and longer branches in nutrient-rich zones (Araya et al. 2016). The root system architecture can be affected by plastic directional growth in response to a directional stimulus, adaptation involving lateral root emergence, and adaptation of the growth rate between the mother root and its lateral roots. These play important roles in the plasticity of the root system architecture when a root system interacts with its surroundings.

To describe the root system architecture in relation to soil heterogeneity, many root system architecture models have been developed, because root systems are difficult, challenging, and costly to observe directly. For example, the first RSA model called ROOTMAP was developed to simulate root system plasticity and proliferation (Diggle 1988). Then, other RSA models (such as RootTyp, SimRoot, ArchiSimple, SPACSYS, R-SWMS, and RootBox) were successively developed to model the root system, root architecture plasticity, and its interaction with soil. Although the above models are similar, they actually have quite different simulation approaches and their own emphases (Dunbabin et al. 2013). Recently, CRootBox and OpenSimRoot were developed to model the root architecture and its interactions with static and dynamic soil environments. They are open source and flexible frameworks for modeling the architecture and its interactions with soil (Postma et al. 2017; Schnepf et al. 2018). All of these RSA models can reproduce root system architectures close to those observed in natural environment based on root growth and development processes. However, these models are all based on mathematical methods, usually statistical models, to generate RSA. Even though these models perfectly reproduce root's architecture, they do not clearly explain decision processes of root growth and development.

Recently, there has been interest in the possibility that a root system can be described in terms of swarming. Previous studies have examined the evidence to support root swarming. For example, Ciszak et al. (2012) presented a theoretical model in terms of swarming and revealed that roots could be influenced by their neighbors to induce a tendency to align the directions of their growth. Matos et al. (2014) used the collective decision of apexes to decide which nutrients to explore. Barlow and Fisahn (2013) described a set of swarming criteria that is generally acceptable for plants and animals. Then, evidence was presented to show that swarming might be a property of roots. McCleery et al. (2017) viewed a root as a swarm of coupled multicellular structures and proposed that root foraging could be regarded as the collective behavior of these structures.

A new focus on swarming behavior in the root system has arisen. Nevertheless, no systematic analysis has been performed to identify the influences of swarming behavior on RSA. Therefore, the objective of our investigation is to propose swarming behavior model and then to analyze plasticity of RSA under a heterogeneous soil environment.

\section{MATERIALS AND METHODS}

\section{Root system architecture model}

ArchiSimple is a simple and generic model in which a root system can be represented as a set of small segments (Pagès et al. 2012). ArchiSimple has also been calibrated and evaluated on a number of species, such as banana, pea, peach tree, cutleaf germander, and maize (Pagès et al. 2014). ArchiSimple is easily connected to a soil model to present the plasticity of the interaction between a root system and the environment. Therefore, ArchiSimple is used as a base to model root growth. In ArchiSimple model, potential RSA is modified by the application of rules (such as root elongation rules and root branching rule). 
In the interaction between root system and soil, nutrient uptake may be less or more demanded of the single root. When demand and absorption of single root are unbalanced, single root growth is stressed. Therefore, we define single root growth stress ( $r g s)$ as follows (Postma et al. 2017):

$$
\operatorname{rgs}(u)=\left\{\begin{array}{rl}
0 & u_{\min } \geq u \\
\frac{u-u_{\min }}{u_{o p t}-u_{\min }} & u_{\min }<u<u_{o p t} \\
1 & u \geq u_{o p t}
\end{array}\right.
$$

where: $u$ is the local resource absorption in each time interval and $u_{\min }$ is the minimal resource demand. In the simulation, $u_{\min }$ is set to zero for simplification and $u_{\text {opt }}$ is the optimal resource demand. Although the optimal resource demand of root will change with the growth time, we simply set $u_{\text {opt }}$ to a constant value in the simulation. The actual RSA is then calculated by multiplying potential RSA and root growth stress ( $r g s)$.

\section{Soil representation}

The approach to soil representation is inspired by the work of Henke et al. (2014). We treat nutrients as a set of very small particles. Within a virtual soil box of $0.25 \mathrm{~m}^{3}(0.5 \mathrm{~m} \times 1 \mathrm{~m} \times 0.5 \mathrm{~m})$, the soil is divided into horizontal layers (thickness: $0.05 \mathrm{~m}$ ). In this article, the root initial radius is set to $0.0018 \mathrm{~m}$ and the root initial position is set at $(0.25$, $0.15,0.25)$. Generally, each horizontal layer has a constant number $(\mathrm{N}=34300)$ of nutrient particles. Three different nutrient particle distributions are considered to analyze the effect of the swarming behavior on root system growth. In a random distribution, $\mathrm{N}$ particles are randomly distributed in each horizontal layer of soil. In the layer distribution, the horizontal layers are divided into four parts. Each part has five horizontal layers. In the first and third parts, $\mathrm{N}$ particles are randomly distributed in each horizontal layer. In the second and fourth parts, $\mathrm{N}^{*} 0.5$ particles are randomly distributed in each horizontal layer. In the gradient distribution, the number of particles $\left(\mathrm{N}^{\prime}\right)$ distributed in each horizontal layer is reduced from top to bottom.
In the simulation, the reduction function is given as follows:

$$
N^{\prime}=\frac{N}{1+\text { layer }_{i} * b}
$$

where layer $_{i}$ is the index of the soil horizontal layer and $b$ is the reduction speed.

\section{Swarming behavior model}

Studies have demonstrated that it is possible to generate behavioral patterns very similar to those of a real swarm by considering the repulsion, alignment, and preference (Couzin 2007; Couzin et al. 2005). In the model, we consider the single root as a moving individual. We treat the length of the single root as the temporal history of the individual. Each single root is assumed to interact with its neighboring single roots, causing spatial alignment or repulsion ( $\mathrm{Li}$ et al. 2016). We suppose that each single root can interact with a neighboring root within two volumes (Fig. 1).

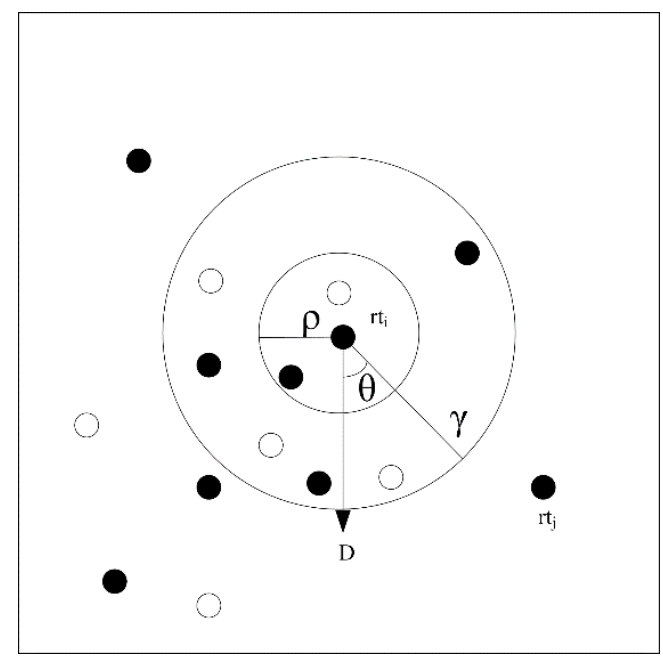

Fig. 1. Schema of neighboring single roots. $\mathrm{rt}_{\mathrm{i}}$ defines the position of the ith single root, $\mathrm{D}$ defines the growth direction of the ith single root, black circles represent single root tips, and white circles represent nutrient particles

We call the inner spherical volume the repulsive volume. The volume involves a single root tip situated at the center of a sphere $\left(\mathrm{rt}_{\mathrm{i}}\right)$ with a constant radius $(\rho)$. The repulsive volume is the minimum distance maintained between the single root tip and another single root tip. A single root tip will turn away from its neighbors within the volume. 
If neighbors are not detected within the repulsive volume, then an aligned volume is constructed, with a single root tip situated at the center of a sphere $\left(\mathrm{rt}_{\mathrm{i}}\right)$ with a constant radius $(\gamma)$. The aligned volume is the social interaction range, within which a single root tip will tend to be aligned with other neighboring single roots (Fig. 1).

Inside the repulsive volume, a single root tip set $\left(\mathrm{C}_{\mathrm{r}}\right)$ can be calculated. Interactions between ith single root tip and jth single root tips in $\mathrm{C}_{\mathrm{r}}$ can be defined as:

$$
\mathrm{D}_{\mathrm{C}}=-\sum_{\mathrm{j} \in \mathrm{C}_{\mathrm{r}}}^{\mathrm{j} \neq \mathrm{i}} \frac{\mathrm{rt}_{\mathrm{j}}-\mathrm{rt}_{\mathrm{i}}}{\left|\mathrm{rt}_{\mathrm{j}}-\mathrm{rt}_{\mathrm{i}}\right|}
$$

where $\mathrm{D}_{\mathrm{C}}$ is the repulsive direction between the ith single root and $\mathrm{C}_{\mathrm{r}}$ and $\mathrm{rt}_{\mathrm{j}}$ defines position of the jth single root.

If neighbors are not detected within the repulsive volume, a single root tip set $\left(\mathrm{A}_{\mathrm{r}}\right)$ within the aligned volume can be calculated. Interactions between ith single root tip and jth single root tip in $A_{r}$ are defined as alignment directions in equation 4.

$$
D_{A}=\sum_{j \in A_{r}}^{j \neq i} \frac{r_{j}-r_{i}}{\left|r_{j}-r_{i}\right|}+\sum_{j \in A_{r}}^{j \neq i} \frac{D_{j}}{\left|D_{j}\right|}
$$

where $\mathrm{D}_{\mathrm{A}}$ is the alignment direction between the ith single root and $A_{r}$ and $D_{j}$ is the growth direction of the jth single root.

Finally, the growth orientation $\left(D_{s}\right)$ of the ith single root tip affected by repulsion and alignment can be obtained as follows:

$$
\mathrm{D}_{\mathrm{S}}= \begin{cases}\frac{\mathrm{D}_{C}}{\left|\mathrm{D}_{C}\right|} & C_{r} \neq \text { null } \\ \frac{\mathrm{D}_{\mathrm{A}}}{\left|\mathrm{D}_{\mathrm{A}}\right|} & C_{r}=\text { null }\end{cases}
$$

\section{Preference affected by nutrient}

The nutrient distribution can affect the preferred growth direction of individual single root, which can be spread by social interactions. We suppose that each single root perceives nutrient particles within sphere-shaped and cone-shaped volumes, as shown in Figure 1. The sphere-shaped volume is the same zone as the repulsive volume because that root tends to monopolize resources in this region.
A cone-shaped volume is situated at $\mathrm{rt}_{\mathrm{i}}$ with the same radius $(\gamma)$ of aligned volume and has a constant opening angle $(\theta)$. When a nutrient particle is positioned in a sphere-shaped volume, this particle is removed from the scene, and its content is added to the local nutrient pool of a single root. When a nutrient particle is positioned in a cone-shaped volume and is not in a sphere-shaped volume, this particle can affect the preferred growth direction ( $p r d$ ) of a single root. It can be calculated that a single root tip turns toward the highest gradient (mean position) of those particles.

The preferred growth direction ( $p r d$ ) shows that a single root can turn toward a greater concentration of available sources. It affects the growth orientation $\left(D_{s}\right)$ of a single root by social interactions as follows:

$D^{\prime}=\frac{D_{S}+\omega^{*} \text { prd }}{\mid D_{S}+\omega^{*} \text { prd } \mid}$

in which $\omega$ is the weighting term between the preferred growth direction and the effect of repulsion and alignment.

In the simulation, $\omega$ is set to one. Individual single roots tends to balance its directions between preference and other directions from social interactions in swarms. At each time interval, the growth direction of a single root affected by the swarming behavior can be calculated using three components (Pagès 2011): its initial direction (D), the effect of the swarming behavior ( $\left.\mathrm{D}^{\prime}\right)$, and the vertical downward component modulated by gravitropism. To comparatively analyze the effects of the swarming behavior on root plasticity, we also present root characteristics with random growth (called nonswarming behavior) in Pagès et al. (2012).

In the research, the number of days for root growth is set to 20 . The set of parameters used for each simulation is presented in Table 1. For each soil scenario (random distribution, layer distribution, and gradient distribution) and each mechanism (swarming behavior mechanism and non-swarming behavior mechanism), five replicate simulations of root system growth were run. Because we study the same root system for two mechanisms, the results are compared at the $5 \%$ probability level based on a repeated measure ANOVA test. 
Table 1. Parameters used in the study

\begin{tabular}{lccl}
\hline Model parameter & Value & Unit & \multicolumn{1}{c}{ Description } \\
\hline Root growth period & 20 & day & Steps of simulation \\
Root initial radius & 0.0018 & $\mathrm{~m}$ & Root radius at the initiation of the root \\
Root initial position & $(0.25,0.15,0.25)$ & - & Root tip position at the initiation of the root \\
$\mathrm{E}$ & 12 & per day & Slope of growth rate versus diameter (Pagès 2011) \\
$\mathrm{d}_{\min }$ & 0.0002 & $\mathrm{~m}$ & Minimum diameter (Pagès 2011) \\
$\mathrm{d}_{\max }$ & 0.0018 & $\mathrm{~m}$ & Maximum diameter (Pagès 2011) \\
ipd & 0.0012 & $\mathrm{~m}$ & Interprimordium distance (Pagès 2011) \\
plre & 0.7 & - & Potential probability of lateral root emergence (Araya et al. 2016) \\
$\sigma$ & 2 & - & Variation in diameter of branch root (Pagès 2011) \\
$\rho$ & 0.01 & $\mathrm{~m}$ & Radius of repulsive volume (Henke et al. 2014) \\
$\gamma$ & 0.05 & $\mathrm{~m}$ & Radius of attractive volume (Henke, et al. 2014) \\
$\theta$ & 65 & $\circ$ & Opening angle of the cone within which the nutrient particle is absorbed (Henke et al. 2014) \\
Gravitropic intensity & 0.15 & - & Intensity of gravitropism (Henke et al. 2014) \\
Intensity of D' & 0.75 & - & Intensity with which the root turns by the swarming behavior (Henke et al. 2014) \\
\hline
\end{tabular}

\section{RESULTS}

\section{Primary root length}

The elongation of the primary root strongly depends on the resource particle distribution (Fig. 2). The longest primary root length is obtained in a random distribution. The lengths of the primary root are $0.5709 \pm 0.0184 \mathrm{~m}$ and $0.5840 \pm 0.0062 \mathrm{~m}$ for the swarming behavior and non-swarming behavior, respectively (Fig. 2a). In the layer distribution, the difference in the primary root length first increases and then decreases when the primary root has crossed into soil layers with fewer resource particles between the swarming behavior and non-swarming behavior (Fig. 2b). In the gradient distribution, the difference in the primary root length continuously increases between the two behaviors (Fig. 2c). Comparing the primary root length in the random, layer, and gradient distributions, significant differences are observed between the swarming behavior and non-swarming behavior in the layer and gradient distributions.

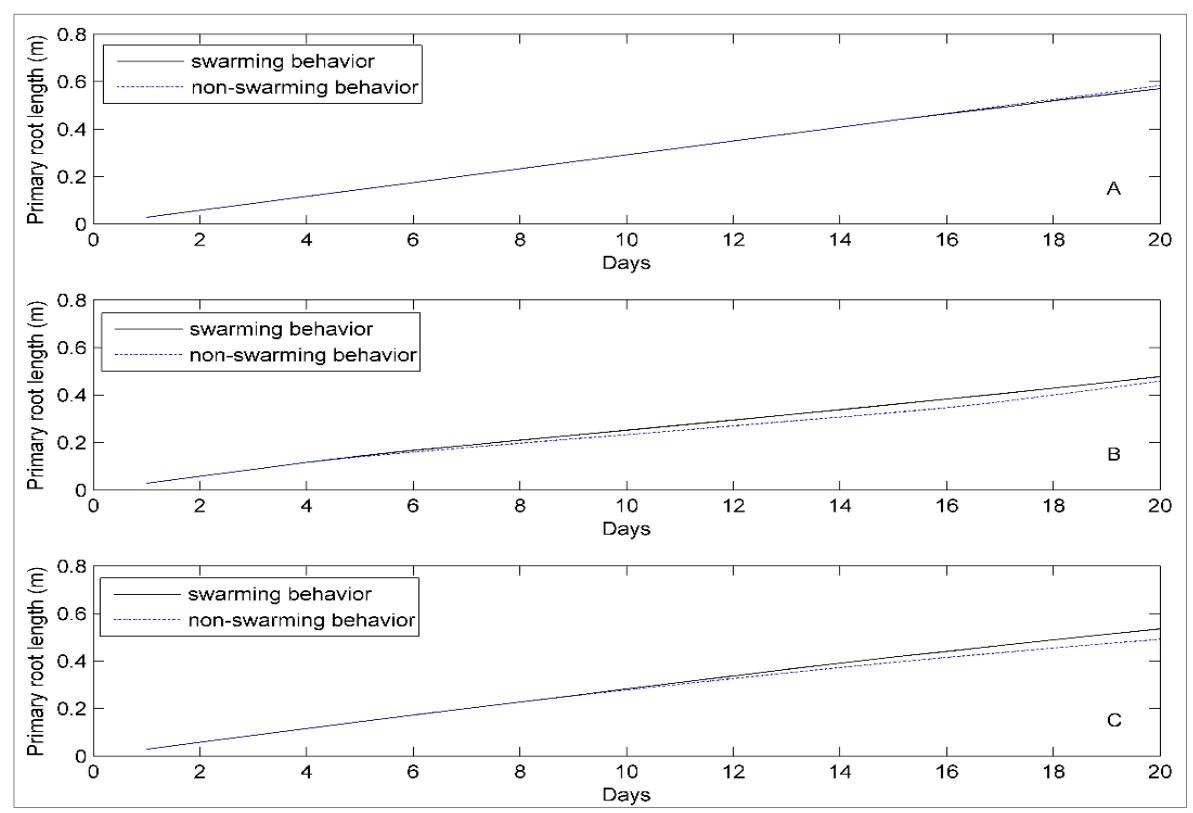

Fig. 2. Effect of swarming behavior on primary root lengths; primary root lengths in (a) random (p=0.174), (b) layer $(\mathrm{p}=0.003)$, and $(\mathrm{c})$ gradient $(\mathrm{p}=0.00)$ distributions 


\section{Lateral root length}

The elongation of the lateral root also strongly depends on the distribution of the resource particles. The greater the number of resource particles distributed in the soil is, the longer is the lateral root length produced. The total lateral root lengths are shorter in the layer and gradient distributions than those in the random distribution (Fig. 3). In the random distribution, the lateral root length of the root system with the swarming behavior is 1.11 times more than that of the system with the non-swarming behavior
(Fig. 3a). The lateral root length of the root system with the swarming behavior is 2.11 times greater than that of the system with the non-swarming behavior (Fig. 3b). In the gradient distribution, the difference in the lateral root length of the root system between the swarming behavior and non-swarming behavior can also be up to 1.13 times (Fig. 3c). Lateral root elongation can be strongly promoted by the swarming behavior. A significant difference is observed between the swarming behavior and nonswarming behavior only in the layer distribution.

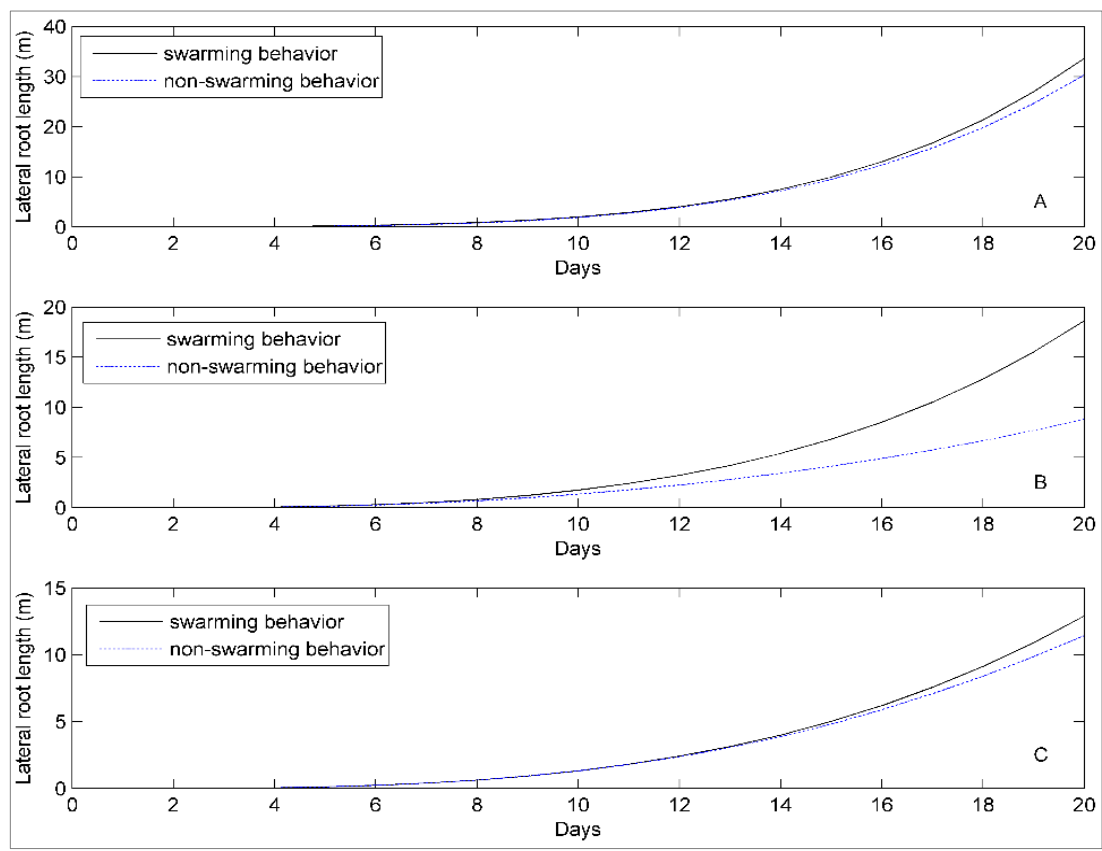

Fig. 3. Effect of the swarming behavior on the lateral root length; lateral root lengths in the (a) random $(\mathrm{p}=0.591)$, (b) layer $(\mathrm{p}=0.00)$, and $(\mathrm{c})$ gradient $(\mathrm{p}=0.491)$ distributions

\section{Lateral root distribution}

We present the lateral root distribution in which the percentage of the lateral root number is expressed at various soil depths (Fig. 4). In the random distribution, $89.66 \%$ of the lateral root number on the primary root is positioned at a depth of $0.0-0.5 \mathrm{~m}$ with the swarming behavior, whereas $66.23 \%$ is positioned in the same zone with the non-swarming behavior (Fig. 4a). In the layer distribution, with the swarming behavior, $50.52 \%$ of the lateral root number on the primary root is positioned at $0.0-0.25 \mathrm{~m}, 47.94 \%$ of the lateral root number on the primary root is positioned at $0.25-$ $0.5 \mathrm{~m}$, and $1.54 \%$ of the lateral root number on the primary root is positioned at $0.5-0.75 \mathrm{~m}$. These results show that lateral root growth on the primary root with the swarming behavior has difficulty in crossing the soil layers with fewer resource particles from 0.25 to $0.5 \mathrm{~m}$ and exploring other soil layers with more resource particles from 0.5 to $0.75 \mathrm{~m}$. When the non-swarming behavior is applied, those values are $33.53 \%, 40 \%$, and $26.47 \%$, respectively. These results show that lateral root growth on the primary root with nonswarming behavior crosses the soil layers with fewer resource particles from 0.25 to $0.5 \mathrm{~m}$ and produces more lateral roots in the soil layers with more resource particles from 0.5 to $0.75 \mathrm{~m}$ (Fig. 4b). 
In the gradient distribution, a higher percentage of the lateral root number on the primary root $(32.38 \%)$ is found with the swarming behavior in the top $0.25 \mathrm{~m}$ and a lower percentage $(26.05 \%)$ is found with the non-swarming behavior. From 0.25 to $0.5 \mathrm{~m}$, those values are $67.62 \%$ and $58.14 \%$, respectively, for the swarming behavior and nonswarming behavior (Fig. 4c). These results show that lateral root growth on the primary root with the swarming behavior is not enough motivation to develop deep soil when individual single roots have explored the topsoil with rich nutrients.

Although the lateral root distribution and root system distribution were computed only at the final time point (20 days), these data were associated with
20 horizontal layers (thickness: $0.05 \mathrm{~m}$ ). The location of each data point of the lateral root distribution and root system distribution could not be changed. These data should be treated as vectors. In this article, the vector cosine angle is used to measure the difference between data of the lateral root distribution.

The vector cosine angles of the lateral root distribution for the swarming behavior and nonswarming behavior were $0.835,0.780$, and 0.797 in the random, layer, and gradient distributions, respectively. We can conclude that the minimum difference in the lateral root distribution was obtained in the random distribution and that the maximum difference in the lateral root distribution was obtained in the layer distribution.

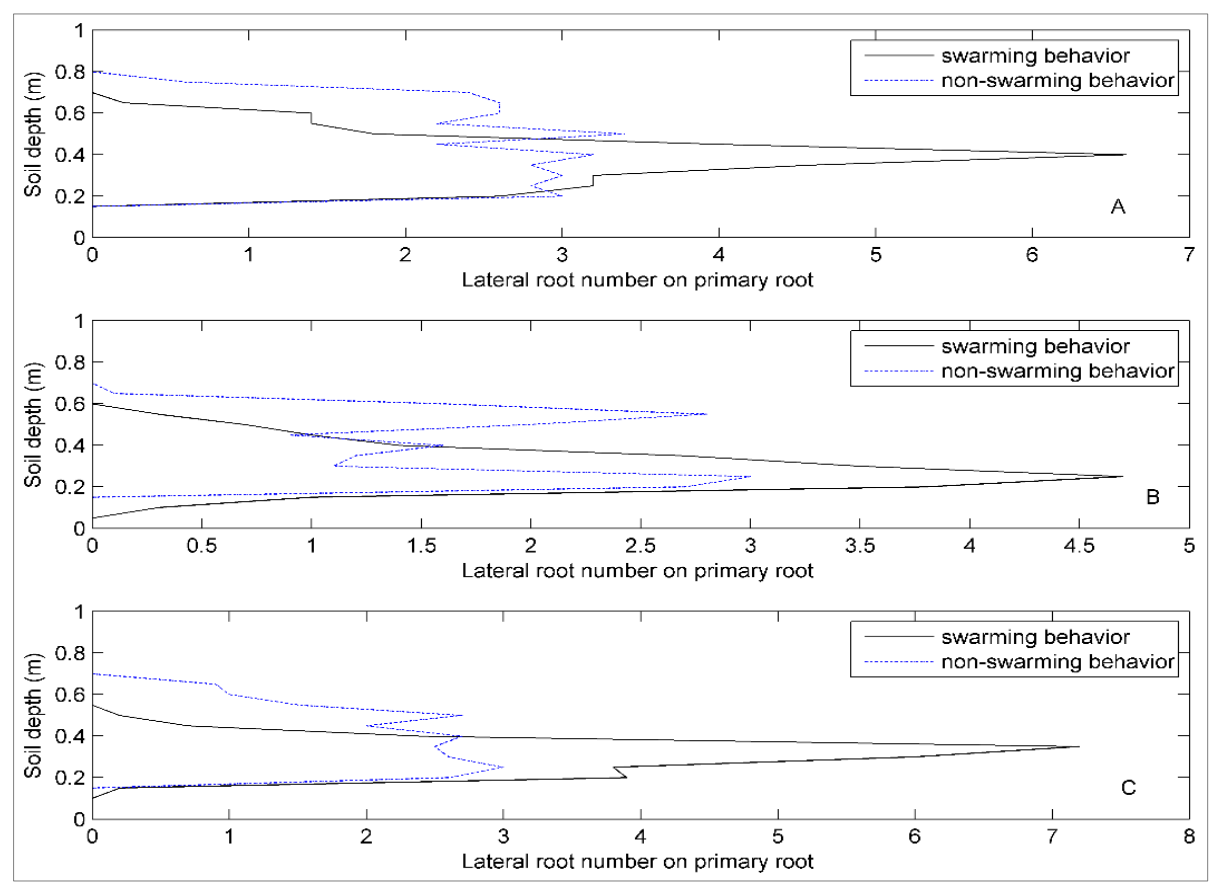

Fig. 4. Effect of swarming behavior on profiles of the lateral root number on the primary root; profiles of the lateral root number on the primary root in the (a) random, (b) layer, and (c) gradient distributions

\section{Root system distribution}

Root profiles where the number of organs at various soil depths is also expressed as a percentage of the total number of root system organs produced (Fig. 5). With the swarming behavior, the maximum depth that the root system can reach is $0.65 \mathrm{~m}$, and $81.6 \%$ of the root system organs are positioned at $0.25-0.5 \mathrm{~m}$. With the non-swarming behavior, the distance can be up to $0.7 \mathrm{~m}$, and $81.21 \%$ of root system organs are positioned at
$0.25-0.5 \mathrm{~m}$ in the random distribution (Fig. 5a). In the layer distribution, the phenomenon of topsoil exploration is obvious. The maximum depth that the root system with the swarming behavior can reach is $0.55 \mathrm{~m}$, and $72.97 \%$ of the root system organs are positioned at $0.0-0.25 \mathrm{~m}$. The maximum depth that the root system with the non-swarming behavior can reach is $0.75 \mathrm{~m}$, and $23.3 \%$ of the root system organs are positioned in the same zone (Fig. 5b). 
In the gradient distribution, the same trend of the root profile is also observed. In the top $0.25 \mathrm{~m}$, $43.73 \%$ of the root system organs can be found for the swarming behavior, whereas $10.63 \%$ of the root system organs can be found for the non-swarming behavior (Fig. 5c). We can see that root growth with the non-swarming behavior leads to deeper soil penetration, whereas root growth with the swarming behavior leads to a shallower soil occupation.
The vector cosine angles of the root system distribution for the swarming behavior and nonswarming behavior are $0.907,0.662$, and 0.740 in the random, layer, and gradient distributions, respectively. The minimum difference in the total lateral root distribution is obtained in the random distribution. The maximum difference in the total lateral root distribution is obtained in the layer distribution.

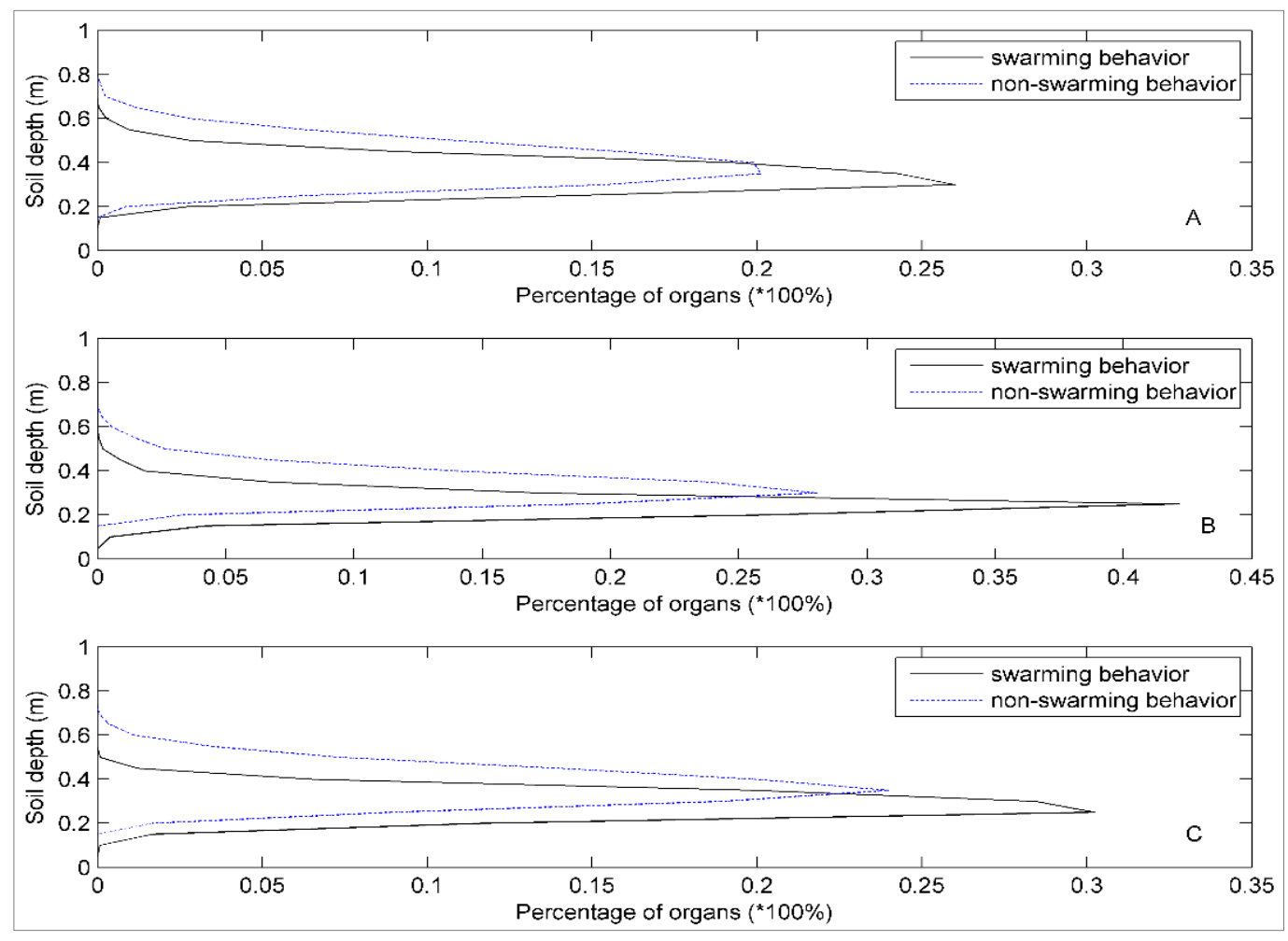

Fig. 5. Effect of swarming behavior on the root system profile; root system profiles in the (a) random, (b) layer, and (c) gradient distributions

\section{Particle uptake}

We present the total particle uptake of the whole root system during the root growth period in Figure 6. In the random distribution, there is a difference in total particle uptake of the whole root system between the swarming behavior (4.58\% uptake) and non-swarming behavior (3.65\% uptake; Fig. 6a). In the layer distribution, there is a significant difference between the swarming behavior (3.32\% uptake) and non-swarming behavior (1.17\% uptake;
Fig. 6b). In the gradient distribution, those values are $2.67 \%$ and $2.04 \%$ for the swarming behavior and non-swarming behavior, respectively (Fig. 6c). Significant differences are observed between the swarming behavior and non-swarming behavior in the layer and gradient distributions. These results show that compared with the nonswarming behavior, the total resource uptake and root foraging ability can be promoted by the swarming behavior. 


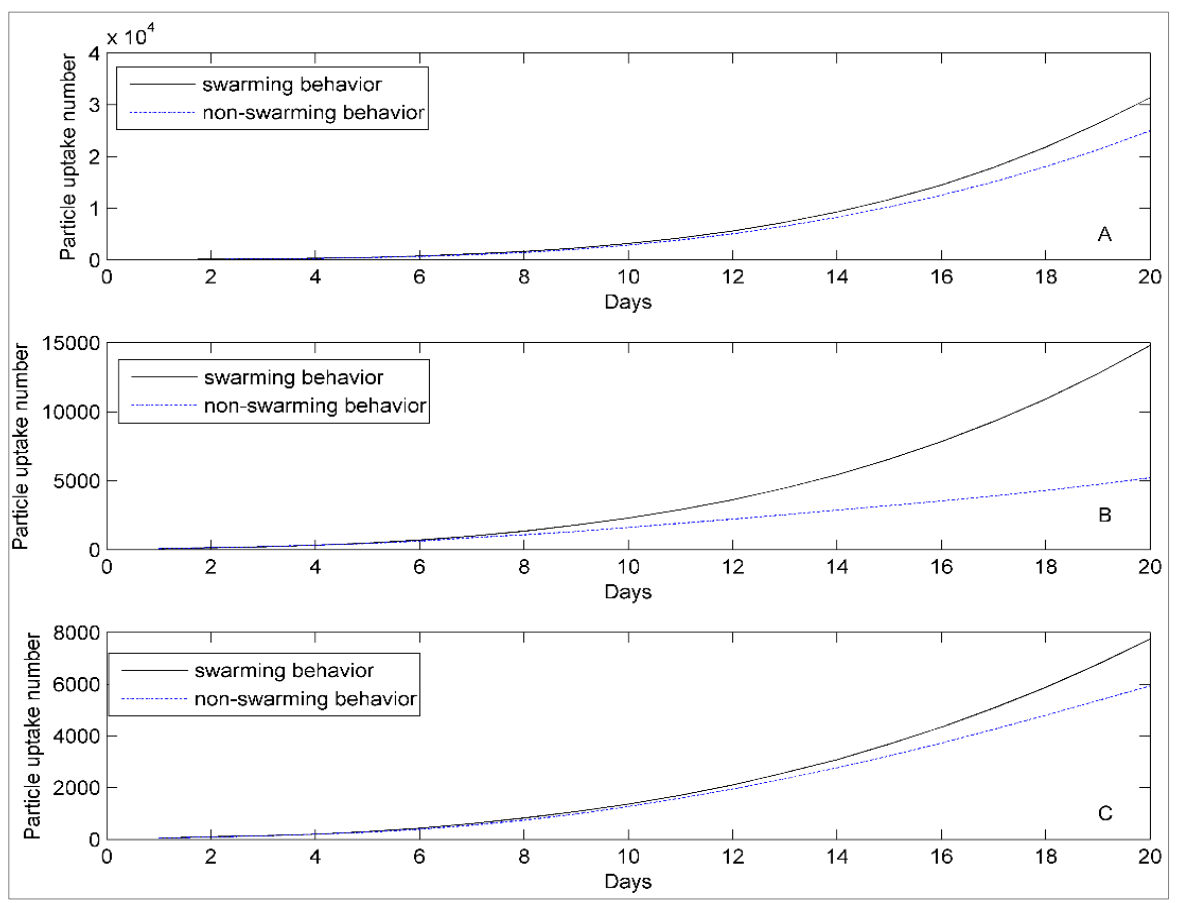

Fig. 6. Effect of swarming behavior on the resource particle uptake; resource particle uptake in the (a) random $(\mathrm{p}=0.069),(\mathrm{b})$ layer $(\mathrm{p}=0.00)$, and $(\mathrm{c})$ gradient $(\mathrm{p}=0.00)$ distributions

\section{Root growth direction}

The effect of the swarming behavior on the root growth directions in the random, layer, and gradient distributions during the root growth period is presented in Figure 7. The results show that the root growth direction can be greatly influenced by the swarming behavior in all three distributions. In the layer distribution, there are more lateral roots in the upper soil, and they grow toward the soil surface (Fig. 7b). In the gradient distribution, this phenomenon is more obvious (Fig. 7c). When a single root senses rich nutrients, the information can be transmitted to some roots by the swarming behavior. As we can see, the growth directions of the root system, including the primary root (thick line) and lateral roots (thin lines), change greatly to carry out exploration of the soil.
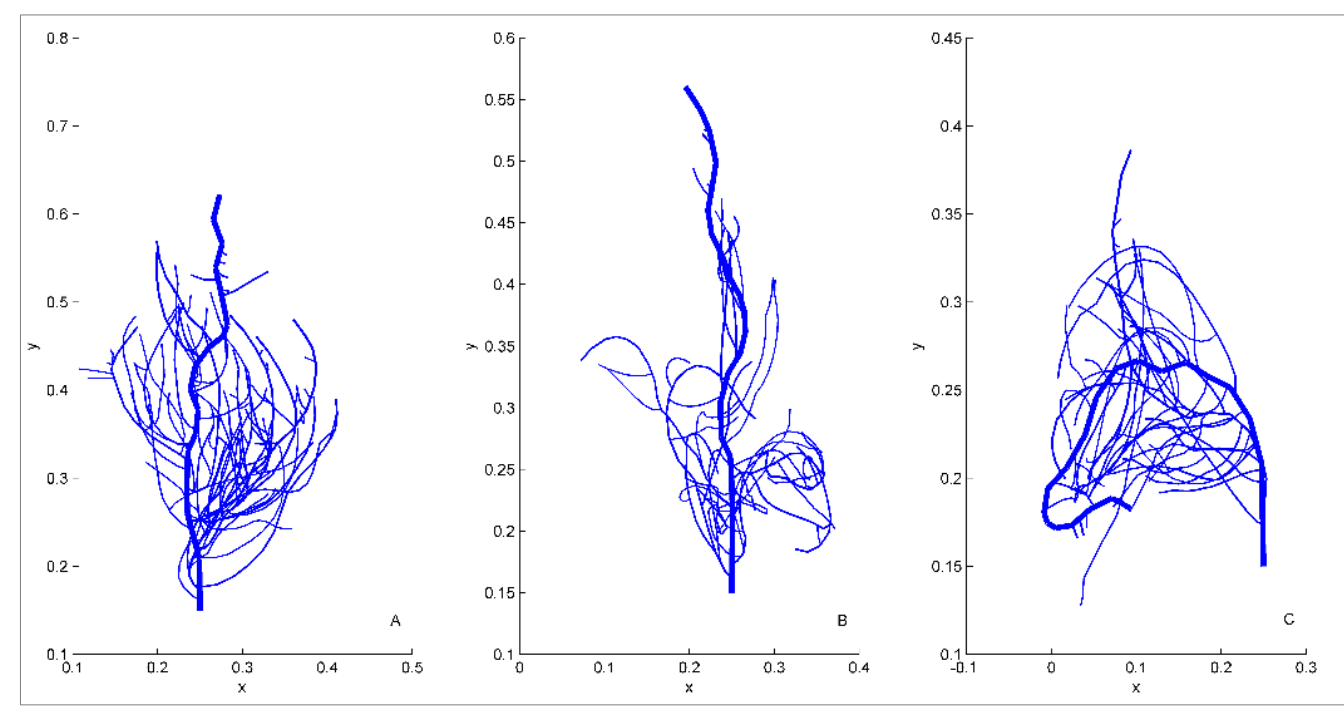

Fig. 7. Effect of swarming behavior on root growth directions; root (radius $>0.001 \mathrm{~m}$ ) growth direction in the (a) random, (b) layer, and (c) gradient distributions 


\section{DISCUSSION}

Roots display complex morphological characteristics because of the diverse growth behaviors of individual structural components that comprise the root system architecture (Araya et al. 2016). To explain the architectural plasticity of the root system, we propose viewing it as a swarm of individual single roots. In this study, we propose a dynamic model to assess the effect of the swarming behavior on root based on hierarchical relationships between the primary and lateral roots by way of simulation.

Compared with the root characteristics under the non-swarming behavior, the primary root length can be promoted by the swarming behavior in the layer and gradient distributions (Fig. 2b, c), and the lateral root length can be significantly promoted by the swarming behavior in the three distributions (Fig. 3). Because the root primordium is initiated by a constant interprimordium distance, the total number of root primordia will be proportional to the total length of the root system. As only a limited proportion of the root primordia can emerge (Lucas et al. 2008), the lateral root emergence can be considered an event that occurs with a potential probability of lateral root emergence affected by root growth stress $(\mathrm{rgs})$. In the simulation, the number of lateral root on the primary root with the swarming behavior increases in nutrient-rich zone (Figs. 4 and 5). The enhancement of the primary root length, and lateral root length, lateral root number on the primary root with the swarming behavior is due to an increase in the foraging ability of the individual single roots (Gleeson et al. 2010), and the total resource particle uptake is promoted (Fig. 6). This can cause an increase in the length of the root segment and a real probability of lateral root appearance. However, in Figures $2 \mathrm{a}$ and $4 \mathrm{a}$, we can see that the final primary root length and lateral root number on the primary root of the root system with the swarming behavior are less than those of the root system with the nonswarming behavior. These results show that the root foraging ability in each iteration rather than the potential growth rate and potential probability of lateral root emergence appears to be the essential factor that differentiates the root-branching and rootgrowing phenotypes (Araya et al. 2016), and root growth with the swarming behavior can improve the root foraging ability, allowing adaptation to heterogeneous soil environments. It has been reported that under heterogeneous soil conditions, emergence of the local lateral root increases in high-nitrate patches, whereas it is suppressed in the surrounding low-nitrate regions (Zhang \& Forde 1998) and in resource-rich soils or soil in which fertilization is applied to the top layer of the soil, shallower growth angles of the axial roots and higher root length density in the upper soil layers have been observed (Manske et al. 2000; Zhu et al. 2005). Our simulation was apparently similar to these phenotypes. In the layer distribution, with the swarming behavior $50.52 \%$ of the lateral root number on the primary root (Fig. 4b) and $72.97 \%$ of the root system organs (Fig. 5b) are positioned at a depth of $0.0-0.25 \mathrm{~m}$. In the gradient distribution, with the swarming behavior $32.38 \%$ of the lateral root number on the primary root (Fig. 4c) and $43.73 \%$ of the root system organs (Fig. 5c) are positioned at a depth of $0.0-0.25 \mathrm{~m}$. These values are all higher than that of the root system with the non-swarming behavior in the same soil zone. These results show that root growth with the swarming behavior can improve the ability to occupy resource-rich soil that has been explored by individual single roots. However, root growth with the swarming behavior also has disadvantages. In the layer distribution, with the swarming behavior $1.54 \%$ of the lateral root number on the primary root (Fig. $4 \mathrm{~b}$ ) and $0.6 \%$ of the root system organs (Fig. 5b) are positioned at $0.5-0.75 \mathrm{~m}$. These values are all smaller than those of the root system with the non-swarming behavior in the same soil zone. There are two resource-rich soil patches at $0.0-0.25$ and $0.5-0.75 \mathrm{~m}$ in the layer distribution. In the gradient distribution, the same phenomenon is found. These results show that root growth with the swarming behavior may inhibit exploration of resource-rich soil that individual single roots do not sense. In the random distribution, the vector cosine angles of the lateral root distribution and root system distribution are 0.835 and 0.907 , respectively. These results show that root growth can be slightly affected by the swarming behavior in the random distribution.

In the gradient distribution, the vector cosine angles of the lateral root distribution and root system 
distribution are 0.797 and 0.740 , respectively. In the layer distribution, these values are 0.780 and 0.662 , respectively. We can see that the vector cosine angles of the lateral root distribution and root system distribution decrease. These results show that root growth can be greatly affected by the swarming behavior in the layer and gradient distributions.

In the swarming behavior model, the parameter radii of the repulsive volume $(\rho)$ and aligned volume $(\gamma)$ have an important impact on root growth. Therefore, we tested the effects of different parameters $(\rho$ and $\gamma)$ on the characteristics of root growth (Appendices A \& B). On the basis of the repeated-measure ANOVA test, the results show that there is no significant difference in root growth characteristics among the $\rho$ values $(\rho=0.01,0.008$, $0.012)$ and $\gamma$ values $(\gamma=0.05,0.04,0.06)$. These results show that small changes in the key parameters ( $\rho$ and $\gamma$ ) do not affect root growth and that the proposed swarming behavior model has robustness.

\section{CONCLUSIONS}

In the current work, we present a dynamic root growth model affected by the swarming behavior based on ArchiSimple. Root foraging and exploration in soil can then be regarded as the collective behavior of individual single roots, and the ability to forage and explore the root system in nutrientrich soil can be promoted by the swarming behavior. Therefore, coordination among individual single roots is particularly important in the root system and root architecture, enabling adaptation to environmental stimuli, and may also be important in the observed complex root architecture. The simulation results show that the swarming behavior may play an important role in the growth of roots. Thus, the swarming mechanism should be considered as a hypothesis for modeling dynamic root growth and development. In the future, theoretical analyses and experimental data should be accumulated to evaluate adaptive plant behavior.

\section{Acknowledgments}

The research is funded by the National Natural Science Foundation of China (No. 61501174), Young Key Teachers Program in Higher Education Institutions from the Henan Educational Committee and Henan University of Engineering (No. 2017GGJS152), Science and Technology Development Program of Henan (No. 162102310399), and Simulation research on mechanism of multi-agent collaborative governance of ecological environment of mid-water source of the South-to-North Water Transfer Project (No. U1704124).

\section{REFERENCES}

Araya T., Kubo T., von Wirén N., Takahashi H. 2016. Statistical modeling of nitrogen-dependent modulation of root system architecture in Arabidopsis thaliana. Journal of Integrative Plant Biology 58(3): 254-265. DOI: 10.1111/jipb.12433.

Barlow P.W., Fisahn J. 2013. Swarms, swarming and entanglements of fungal hyphae and of plant roots. Communicative and Integrative Biology 6(5); e25299, 16 p. DOI: 10.4161/cib.25299.

Cassab G.I., Eapen D., Campos M.E. 2013. Root hydrotropism: An update. American Journal of Botany 100(1): 14-24. DOI: 10.3732/ajb.1200306.

Ciszak M., Comparini D., Mazzolai B., Baluska F., Arecchi F.T., Vicsek T., Mancuso S. 2012. Swarming behavior in plant roots. PLoS One 7(1); e29759, 7 p. DOI: 10.1371/journal.pone.0029759.

Couzin I. 2007. Collective minds. Nature 445(7129): 715 DOI: $10.1038 / 445715 a$

Couzin I.D., Krause J., Franks N.R., Levin S.A. 2005. Effective leadership and decision-making in animal groups on the move. Nature 433(7025): 513-516. DOI: $10.1038 /$ nature 03236.

Diggle A.J. 1988. ROOTMAP - a model in three-dimensional coordinates of the growth and structure of fibrous root systems. Plant and Soil 105(2): 169-178. DOI: $10.1007 /$ bf02376780.

Dunbabin V.M., Postma J.A., Schnepf A., Pagès L., Javaux M., Wu L. et al. 2013. Modelling root-soil interactions using three-dimensional models of root growth, architecture and function. Plant and Soil 372(1-2): 93-124. DOI: 10.1007/s11104-013-1769-y.

Giehl R.F.H., Lima J.E., von Wirén N. 2012. Localized iron supply triggers lateral root elongation in Arabidopsis by altering the AUX1-mediated auxin distribution. Plant Cell 24(1): 33-49. DOI: 10.1105/tpc. 111.092973 .

Gilroy S. 2008. Plant tropisms. Current Biology 18(7): R275-R277. DOI: 10.1016/j.cub.2008.02.033. 
Gleeson S.K., Good R.E. 2010. Root growth response to water and nutrients in the New Jersey Pinelands. Canadian Journal of Forest Research 40(1): 167172. DOI: $10.1139 / x 09-180$.

Henke M., Sarlikioti V., Kurth W., Buck-Sorlin G.H., Pagès L. 2014. Exploring root developmental plasticity to nitrogen with a three-dimensional architectural model. Plant and Soil 385(1-2): 49-62. DOI: 10.1007/s11104-014-2221-7.

Hodge A. 2004. The plastic plant: root responses to heterogeneous supplies of nutrients. New Phytologist 162(1): 9-24. DOI: 10.1111/j.14698137.2004.01015.x.

Kiba T., Krapp A. 2016. Plant nitrogen acquisition under low availability: Regulation of uptake and root architecture. Plant and Cell Physiology 57(4): 707714. DOI: $10.1093 / \mathrm{pcp} / \mathrm{pcw} 052$.

Li S., Liu X., Wang M., Yu W. 2016. Exploring root plasticity to resource patches based on swarm behavior. Acta Physiologiae Plantarum 38(8): 192. DOI: 10.1007/s11738-016-2227-2.

Lucas M., Guédon Y., Jay-Allemand C., Godin C., Laplaze L. 2008. An auxin transport-based model of root branching in Arabidopsis thaliana. PLoS One 3(11); e3673, 13 p. DOI: 10.1371/journal.pone.0003673.

Manske G.G.B., Ortiz-Monasterio J.I., Van Ginkel M., González R.M., Rajaram S., Molina E., Vlek P.L.G. 2000. Traits associated with improved Puptake efficiency in CIMMYT's semidwarf spring bread wheat grown on an acid Andisol in Mexico. Plant and Soil 221(2): 189-204. DOI: 10.1023/a:1004727201568.

Matos T., Cruz C., Correia L. 2014. Root growth model based on swarm intelligence. In: Morte A., Varma A. (Eds.), Root Engineering. Soil Biology 40: 5773. DOI: 10.1007/978-3-642-54276-3_4.

McCleery W.T., Mohd-Radzman N.A., Grieneisen V.A. 2017. Root branching plasticity: collective decisionmaking results from local and global signalling. Current Opinion in Cell Biology 44: 51-58. DOI: 10.1016/j.ceb.2017.03.001.
Pagès L. 2011. Links between root developmental traits and foraging performance. Plant, Cell and Environment 34: 1749-1760. DOI: 10.1111/j.13653040.2011.02371.x.

Pagès L., Moreau D., Sarlikioti V., Boukcim H., Nguyen C. 2012. ArchiSimple: a parsimonious model of the root system architecture. IEEE 4th International Symposium on Plant Growth Modeling, Simulation, Visualization and Applications, pp. 297-303. DOI: 10.1109/pma.2012.6524849.

Pagès L., Bécel C., Boukcim H., Moreau D., Nguyen C., Voisin A.-S. 2014. Calibration and evaluation of ArchiSimple, a simple model of root system architecture. Ecological Modelling 290: 76-84. DOI: 10.1016/j.ecolmodel.2013.11.014.

Postma J.A., Kuppe C., Owen M.R., Mellor N., Griffiths M., Bennett M.J. et al. 2017. OpenSimRoot: widening the scope and application of root architectural models. New Phytologist 215(3): 1274-1286. DOI: 10.1111/nph.14641.

Rellán-Álvarez R., Lobet G., Dinneny J.R. 2016. Environmental control of root system biology. Annual Review of Plant Biology 67(1): 619-642. DOI: 10.1146/annurev-arplant-043015-111848.

Schnepf A., Leitner D., Landl M., Lobet G., Mai T.H., Morandage S. et al. 2018. CRootBox: A StructuralFunctional Modelling Framework For Root Systems. Annals of Botany 121(5): 1033-1053. DOI: 10.1093/aob/mcx221.

Tian H., De Smet I., Ding Z. 2014. Shaping a root system: regulating lateral versus primary root growth. Trends in Plant Science 19(7): 426-431. DOI: 10.1016/j.tplants.2014.01.007.

Zhang H., Forde B.G. 1998. An Arabidopsis MADS box gene that controls nutrient-induced changes in root architecture. Science 279(5349): 407-409. DOI: 10.1126/science.279.5349.407.

Zhu J., Kaeppler S.M., Lynch J.P. 2005. Mapping of QTLs for lateral root branching and length in maize (Zea mays L.) under differential phosphorus supply. Theoretical and Applied Genetics 111(4): 688-695. DOI: $10.1007 / \mathrm{s} 00122-005-2051-3$. 